\title{
PHENETIC AND GENETIC CHARACTERIZATION OF SELECTED ECONOMICALLY IMPORTANT SPECIES IN THE FAMILY ZINGIBERACEAE
}

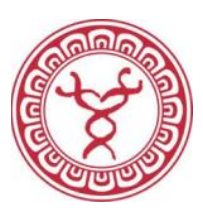

Silva, A. B. W. R ${ }^{1}$, Herath, H. ${ }^{{ }^{*}}$, Senanayake, ${ }^{1}$ S. P., Swarnathilaka, D. B. R².

${ }^{1}$ Department of Botany, University of Kelaniya, Kelaniya, Sri Lanka

2 Plant Production and Tissue Culture Research Centre, Department of Export Agriculture, Walpita, Sri Lanka

\begin{abstract}
Zingiberaceae, includes medicinally and economically important monocotyledons mostly distributed in South and South East Asia. A complete taxonomic knowledge of this plant group in Sri Lanka is lacking hence the present study was carried out to characterize and infer phenetic and genetic relationships of eight economically important zingibers. Morphological and anatomical characters of leaves, rhizomes and adventitious roots and phenolic characters of leaves were cluster analysed using MINITAB 17 to derive relationships. A phylogram was obtained from MEGA software using rDNA ITS sequences amplified by PCR from leaf genomic DNA. Costus speciosus was used as the out group in the phylogenetic study. As per the principal component analysis plant height, rhizome diameter, length of rhizome internodes, leaf length, leaf width and length of leaf petiole can be recommended as suitable for characterization of these taxa. rDNA ITS regions may well be used for Zingiber and Curcuma species identification. Combination of morphological, anatomical and phenolic characters can be used in delimiting commonly used $Z$. officinale cultivars and Curcuma species in Sri Lanka. Further, derived genetic relationships of these taxa would provide better information in characterization of commonly used Zingiber cultivars, Curcuma and Alpinia species in Sri Lanka at the initial developmental stages and would support in quality improvement breeding programs.
\end{abstract}

Key words: Ginger family, ITS, TLC, Zingiberaceae

*Corresponding author: E-mail: harshi@kln.ac.lk

https://orcid.org/0000-0001-8387-0420

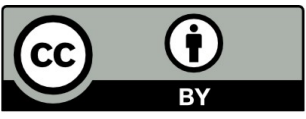

This is an open-access article distributed under the terms of the Creative Commons Attribution License, which permits unrestricted use, distribution and reproduction in any medium, provided the original author and source are credited. 


\section{Introduction}

Members of family Zingiberaceae, commonly known as gingers, are an important group of monocotyledons with considerable economic and ornamental potentials (Uma and Muthukumar, 2014). Most often used gingers belong to the genera Alpinia, Amomum, Curcuma and Zingiber and to lesser extent Boesenbergia, Kaempferia, Elettaria, Elettariopsis, Etlingera and Hedychium (Jatoi et al., 2007). Accurate characterization of taxa is important for their identification, conservation, cultivar development and phylogenetic studies. Characterization of morphological traits and two chloroplast gene regions (trnLtrnF and trnS-trnfM) in Alpinia and Amomum genera have revealed the segregation of species in family Zingiberaceae, a group of Amomum with a new character combination and a new addition to genus Alpinia (A. zerumbet) in Sri Lanka (Karunarathne et al., 2014). Kress et al., (2002), have realigned genera of Zigiberaceae using nuclear Internal Transcribed Spacer (ITS) and chloroplast matK regions. In this research, eight economically important members of family Zingiberaceae; three Zingiber officinale cultivars ('Local', 'Rangun', 'Chinese') two Curcuma species ( $C$. longa, $C$. amada), two Alpinia species ( $A$. purpurata, $A$. calcarata) and Elettaria cardamomum were studied. The objective of this research was to study morphological, anatomical and molecular diversity of selected taxa and to determine their phylogenetic relationships using Costus speciosus (Family Costaceae) as the out group taxon. Family Costaceae is the sister clade to the family Zingiberaceae (Kress et al., 2002).

\section{Material and Methods}

\subsection{Plant material}

The eight Zingiberaceae taxa and $C$. speciosus were collected from the Plant Production and Tissue Culture Research
Centre, Department of Export Agriculture, Walpita and Botanical garden in the University of Kelaniya.

\subsection{Morphological and anatomical studies}

Morphological features of leaves and rhizomes of each taxon were studied in nine replicates. Three plants were studied for each taxon, three replicate values were taken from each plant and the mean value was obtained for each of the quantitative character (Gevu et al., 2014; Uma and Muthukumar, 2014). Floral characters were not studied as no flowers were bloomed during the study period as those are seasonal flowering plants. Vascular bundle arrangement in the leaf petiole and adventitious roots, and stomatal structure were studied by observations of the anatomical sections under the light microscope (Olympus cover 015, Japan; 10x40) and photomicrographs were prepared. All multistate characters were transformed into binary states and a data matrix was prepared. Phenetic relationships of taxa were studied based on the cluster analysis performed using MINITAB 17 software and presented as phenograms. Principal Component Analysis (PCA) on a correlation matrix was used as an objective method to identify clustering patterns of characters, by using MINITAB 17 software.

\subsection{Chemical analysis}

Fresh leaves (2 g) were extracted using methanol $(5 \mathrm{ml})$ and hexane $(5 \mathrm{ml})$ by keeping overnight separately. The crude extracts were used for separation and identification of phenols using silica gel Thin Layer Chromatography (TLC) using BAW (Butanol: Acetic acid: Water, 4:1:5) and $15 \%$ acetic acid (Acetic acid: Water, 15:85) as solvent systems. Plates were dried and observed under ultraviolet (UV) light at $366 \mathrm{~nm}$ wave length in a UV trans illuminator (CAMAG, USA) for spot detection (Karunaratne, 2007). Retardation factor $\left(\mathrm{R}_{\mathrm{f}}\right)$ was calculated for all spots and classified to compound types 
using $\mathrm{R}_{\mathrm{f}}$ value and spot color (Harborne, 1998).

\subsection{Genetic analysis}

Genomic DNA was extracted from about 1 $\mathrm{g}$ of fresh, young leaves of different Zingiberaceae taxa using Cetyl trimethylammonium Bromide (CTAB) based method (Devi et al., 2013). rDNA ITS (Table 1) were amplified from genomic DNA by PCR (GoTaq flexi, Promega Inc, USA) in a thermal cycler (Veriti, Applied Biosystems, USA) at $55^{\circ} \mathrm{C}$ annealing temperature. PCR products were visualized in $1.0 \%$ agarose gels under UV light in a gel documentation system (Quantum, Germany).

Putative ITS regions were sequenced by Sanger method at the Genetech, Colombo. Sequences were edited using BioEdit version 7.2.5. Regions of similarities between sequences were searched by using online National Center for Biotechnology Information, Basic Local Alianment Search Tool(NCBI BLAST: blastn). Phylograms were constructed by MEGA version 7.0.21 using ITS sequences. ITS sequences obtained in the present study and reference sequences obtained from GenBank (NCBI) were used for phylogenetic analysis. The evolutionary history was inferred by Maximum Likelihood method based on the Kimura 2-parameter model. Bootstrap analyses were performed with 1000 replicates. Costus speciosus was used as the out group taxon.

Table 1. ITS primers used in the study

\begin{tabular}{lc}
\hline \multicolumn{1}{c}{ Primer } & \multicolumn{1}{c}{ Sequence (5'-3') } \\
\hline ITS 1 & TCCGTAGGTGAACCTTGCGG \\
ITS 4 & TCCTCCGCTTATTGATATGC \\
\hline
\end{tabular}

\section{Results}

Morphological and anatomical characters of leaves, rhizomes and adventitious roots were observed and recorded for each taxon (Tables 2 and 3). Paratetracytic stomatal type was observed in $E$. cardomomum while all the other taxa have paracytic type of leaf stomata (Fig. $1 \mathrm{~A}$ and 1B). All eight studied taxa of family Zingiberaceae showed ' $U$ ' shape free bundle arrangement of vascular bundles in observed cross sections of leaf petioles except in $C$. speciosus which had the arrangement of flat arc with rib traces (Fig. 1C and 1D). According to the arrangement of vascular bundles in adventitious roots all taxa including the out group have showed radial arrangement of vascular bundles (Fig. 1E).

The cladogram constructed using 18 morphological and anatomical characters of eight taxa indicated two main different evolutionary lines and they have shared a common ancestor (Fig. 2). Z. officinale 'Local' and 'Chines' were clustered into one clade with $100 \%$ similarity. $Z$. officinale 'Local', 'Chinese' and 'Rangun' clustered together with $64.64 \%$ similarity. $C$. longa and $C$. amada were clustered into another clade with $44.10 \%$ similarity. $C$. speciosus represented a separate evolutionary line.

According to the loading plot of PCA (Fig. $3)$, some characters overlapped each other showing the affinity of their influence in deriving relationships and characterization of taxa. According to the PCA plant height, rhizome diameter, rhizome internode length, leaf length, leaf width and leaf petiole length can be considered as more suitable in characterization of these taxa. 
Seven different compound types were identified from the hexane and methanolic leaf extracts in BAW using silica gel TLC (Table 4). In the cladogram (Fig. 4), Z. officinale 'Local' and 'Rangun' were clustered into one clade with $100 \%$ similarity. C. longa and A. calcarata were clustered into another clade with $62.20 \%$ similarity.
The 260:280 and 260:230 ratios of extracted genomic DNA were 1.05-1.13 and 1.05-1.06 respectively, indicating the presence of proteins, EDTA or carbohydrates. PCR products of putative ITS (700-750 bp) were obtained for seven out of nine taxa. Putative ITS sequences of C. longa, C. amada and Z. officinale 'Rangun' retrieved respective species in a blastn with $100-77 \%$ identities (Fig 6).

Table 2: Character comparison chart for morphological and anatomical characters

\begin{tabular}{lll}
\hline \multicolumn{1}{c}{ Character } & \multicolumn{1}{c}{ Character state 0 } & \multicolumn{1}{c}{ Character state 1} \\
\hline Type of plant & Shrub & Herb \\
Plant height & More than $50 \mathrm{~cm}$ & Less or equal than $50 \mathrm{~cm}$ \\
Stem diameter & More than $2.5 \mathrm{~cm}$ & Less or equal than $2.5 \mathrm{~cm}$ \\
Number of internodes & More than 5 & Less or equal than 5 \\
Length of internodes & More than $4.5 \mathrm{~cm}$ & Less or equal than $4.5 \mathrm{~cm}$ \\
Number of leaves & More than 5 & Less or equal than 5 \\
Leaf arrangement & Spiral & Alternate \\
Leaf shape & Ovate & Eliptic \\
Leaf margin & Entire & Ciliate \\
Leaf apex & Attenuate & Acute \\
Leafbase & Obtuse & Acute \\
Leaf venation & Pinnate & Palmate-Parallel \\
Leaflength & More than $20 \mathrm{~cm}$ & Less or equal than $20 \mathrm{~cm}$ \\
Leaf width & More than $4 \mathrm{~cm}$ & Less or equal than $4 \mathrm{~cm}$ \\
Length of leaf petiole & More than $0.5 \mathrm{~cm}$ & Less or equal than $0.5 \mathrm{~cm}$ \\
Leafteeth & Absent & Present \\
Arrangement in vascular & Flat arc with rib trace & U-shape \\
bundles in leaf petiole & & \\
Shape of subsidiary cells in & Paracytic & Paratetracytic \\
leaf stomata & & \\
\hline
\end{tabular}

Table 3: Data matrix for morphological and anatomical characters

\begin{tabular}{|c|c|c|c|c|c|c|c|c|c|}
\hline \multirow{2}{*}{ Character } & \multicolumn{9}{|c|}{ OTU } \\
\hline & 1 & 2 & 3 & 4 & 5 & 6 & 7 & 8 & 9 \\
\hline 1. Habitat & 1 & 1 & 1 & 1 & 1 & 1 & 1 & 1 & 1 \\
\hline 2. Type of plant & 1 & 1 & 1 & 1 & 1 & 0 & 1 & 1 & 1 \\
\hline 3. Plant height & 1 & 1 & 1 & 1 & 1 & 0 & 0 & 1 & 1 \\
\hline 4. Stem diameter & 1 & 0 & 1 & 1 & 1 & 0 & 1 & 1 & 0 \\
\hline 5. Number of leaves & 0 & 0 & 0 & 1 & 1 & 1 & 0 & 1 & 0 \\
\hline 6. Leaf arrangement (Phyllotaxy) & 1 & 1 & 1 & 1 & 1 & 1 & 1 & 1 & 0 \\
\hline 7. Leaf structure & 1 & 1 & 1 & 1 & 1 & 1 & 1 & 1 & 1 \\
\hline 8. Leaf shape & 1 & 1 & 1 & 0 & 0 & 1 & 1 & 1 & 0 \\
\hline 9. Leaf margin & 1 & 1 & 1 & 0 & 0 & 1 & 1 & 1 & 0 \\
\hline 10. Leaf apex & 1 & 1 & 1 & 1 & 1 & 1 & 1 & 1 & 1 \\
\hline 11. Leaf base & 1 & 1 & 1 & 1 & 1 & 1 & 1 & 1 & 1 \\
\hline 12. Leaf venation & 1 & 1 & 1 & 1 & 1 & 1 & 1 & 1 & 0 \\
\hline 13. Leaf length & 1 & 1 & 1 & 0 & 1 & 0 & 0 & 0 & 1 \\
\hline 14. Leaf width & 1 & 1 & 1 & 0 & 0 & 0 & 0 & 1 & 0 \\
\hline 15. Length of leaf petiole & 1 & 1 & 1 & 0 & 0 & 0 & 0 & 1 & 1 \\
\hline 16. Leaf teeth & 1 & 1 & 1 & 0 & 0 & 1 & 1 & 1 & 0 \\
\hline $\begin{array}{l}\text { 17. Arrangement in vascular bundles } \\
\text { in leaf petiole }\end{array}$ & 1 & 1 & 1 & 1 & 1 & 1 & 1 & 1 & 0 \\
\hline $\begin{array}{l}\text { 18. Shape of subsidiary cells in leaf } \\
\text { stomata }\end{array}$ & 1 & 1 & 1 & 1 & 1 & 0 & 1 & 1 & 1 \\
\hline
\end{tabular}




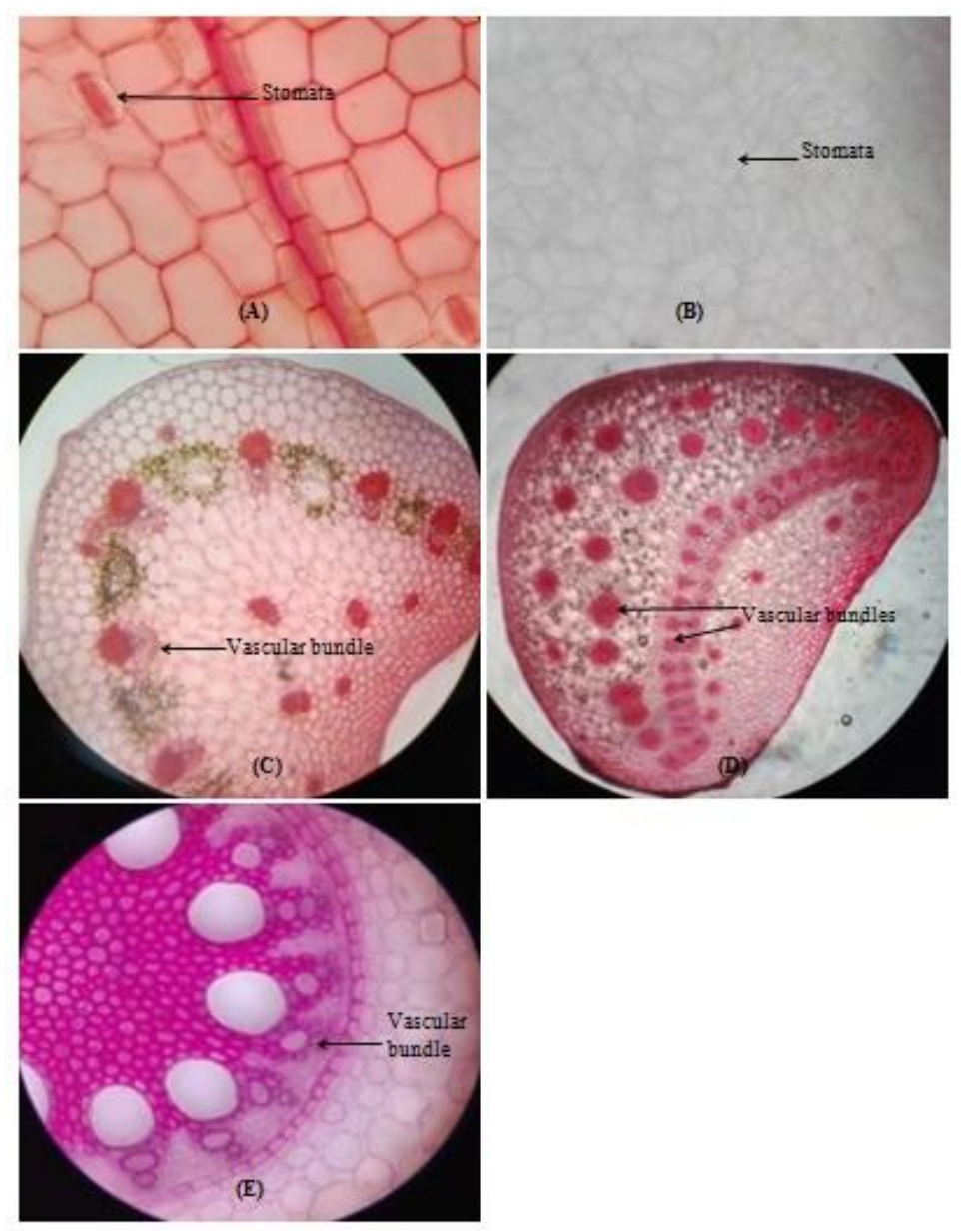

Figure 1: (A) Z. officinale 'Chinese' paracytic stomatal type in upper leaf epidermis (B) $E$. cardomomum paratetracytic stomatal type (C) U - shape free vascular bundle arrangement in $Z$. officinale 'Chinese' leaf petiole (D) Flat arc with rib traces vascular arrangement in $C$. speciosus and (E) Radial vascular arrangement in A. calcarata adventitious root (10 x 40).

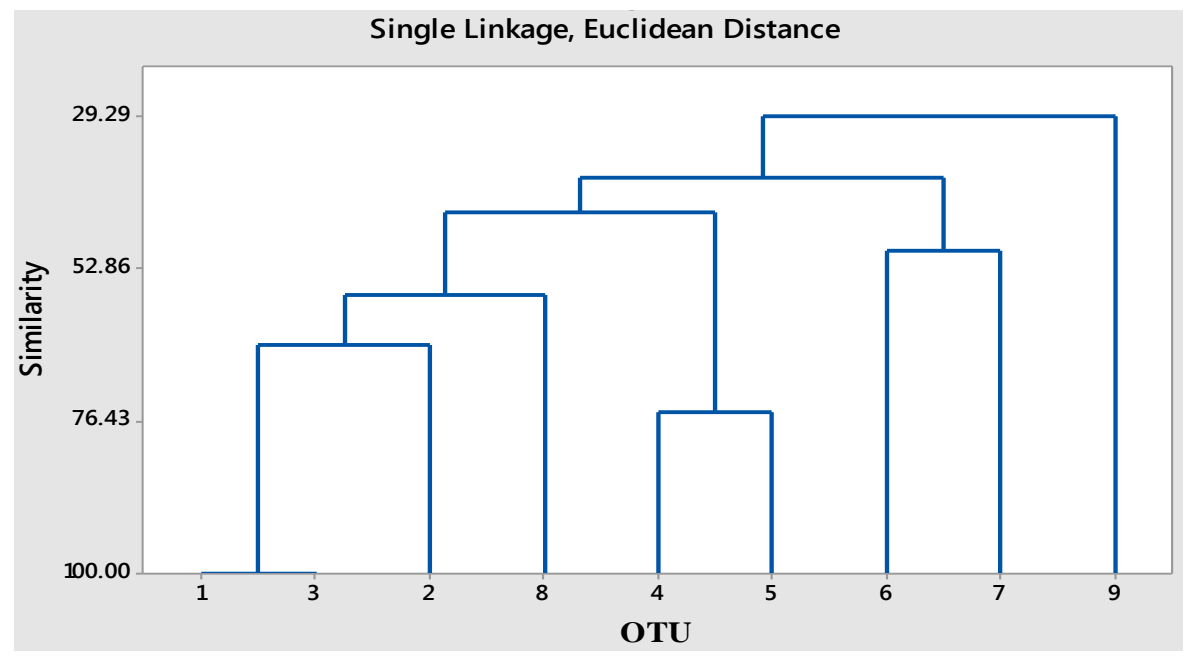

Figure 2: Cladogram constructed for 18 morphological and anatomical characters. OUT 1: Z. officinale 'Local', OUT 2: Z. officinale 'Rangun', OUT 3: Z. officinale 'Chinese', OUT 4: C. longa, OUT 5: C. amada, OUT 6: $E$. cardomomum, OUT 7: A. purpurata, OUT 8: A. calcarata, OUT 9: C. speciosus 


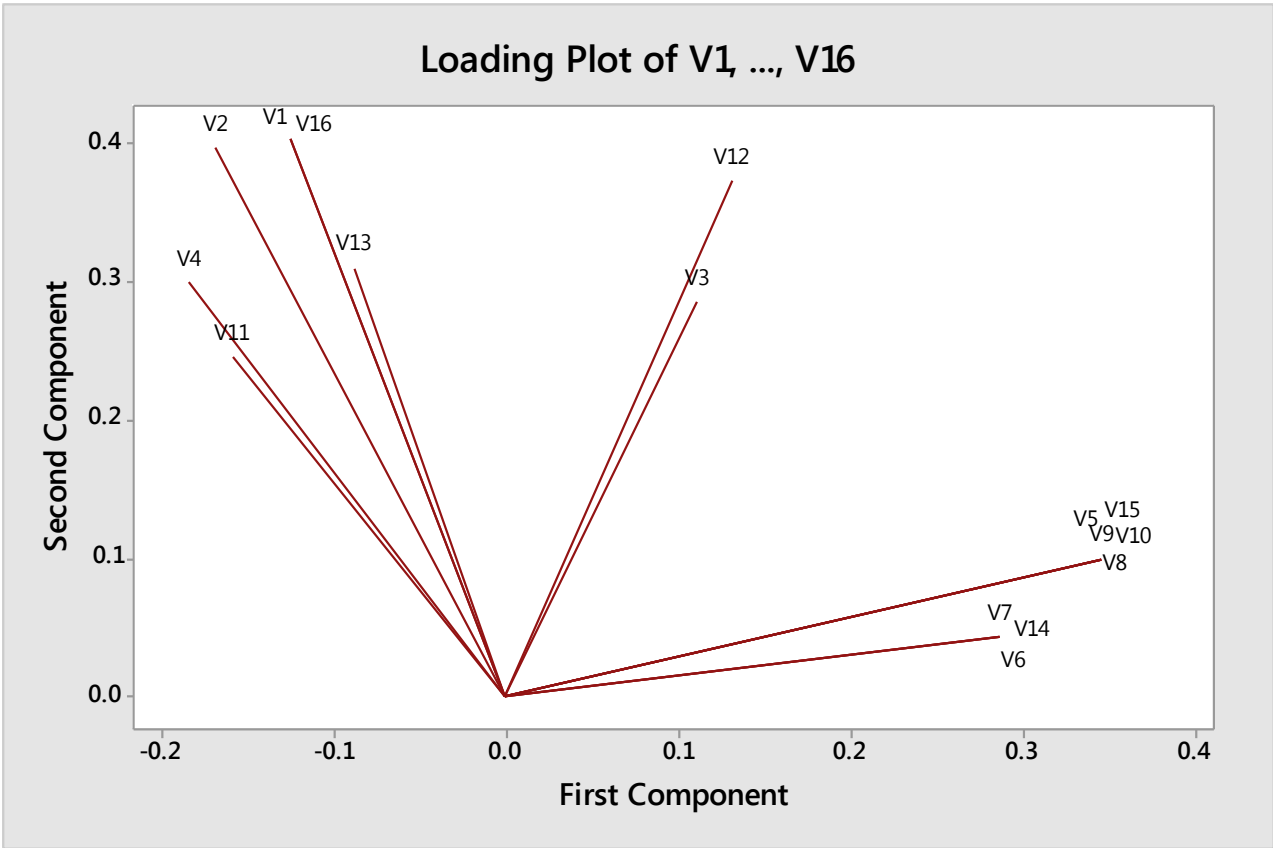

Figure 3: Loading plot of PCA of morphological and anatomical characters of taxa. V1: Type of plant, V2: Plant height, V3: stem diameter, V4: Rhizome internode length, V5: Leaf arrangement, V6: Leaf shape, V7: Leaf margin, V8: Leaf apex, V9: Leaf base, V10: Leaf venation, V11: Leaf length, V12: Leaf width, V13: Leaf petiole length, V14: Leaf teeth, V15: Vascular bundle arrangement in leaf petiole, V16: Shape of subsidiary cells in leaves.

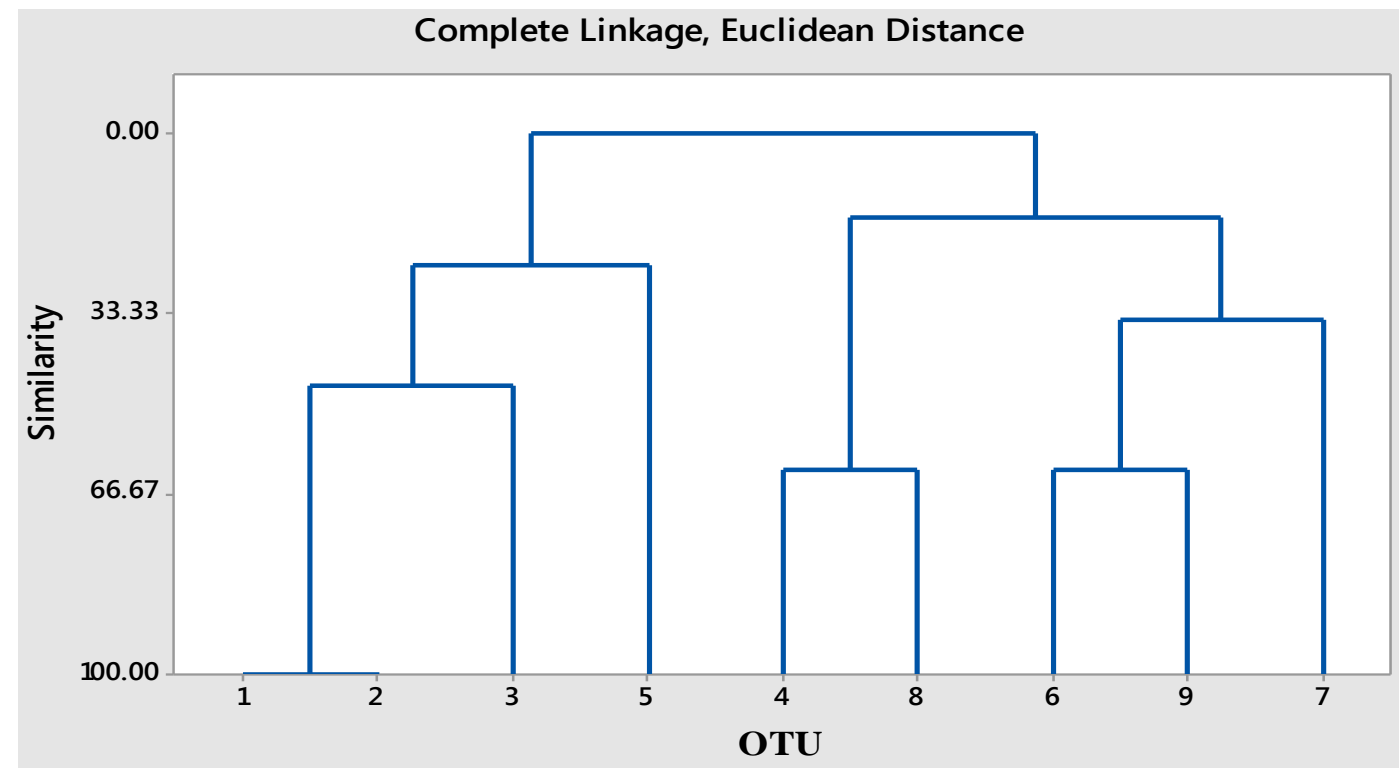

Figure 4: Cladogram constructed for phenolic compounds of leaf extracts. OUT 1: Z. officinale 'Local', OUT 2: Z. officinale 'Rangun', OUT 3: $Z$. officinale 'Chinese', OUT 4: $C$. longa, OUT 5: $C$. amada, OUT 6: E. cardomomum, OUT 7: A. purpurata, OUT 8: A. calcarata, OUT 9: C. speciosus 
Table 4: Seven different compound types separated from hexane and methanol leaf extracts in TLC

\begin{tabular}{ccl}
\hline \multirow{2}{*}{ Compound type } & \multicolumn{2}{c}{ Butanol: Acetic acid: Water (4:1:5) } \\
\cline { 2 - 3 } & $\mathbf{R}_{\mathbf{f}}$ value range & \multicolumn{1}{c}{ Spot colour } \\
\hline 1 & $15-25$ & Brown \\
3 & $26-35$ & Brownish yellow \\
4 & $40-50$ & Brown \\
5 & $51-60$ & Light yellow \\
6 & $61-70$ & Light yellow \\
7 & $71-80$ & Light brown \\
\hline
\end{tabular}

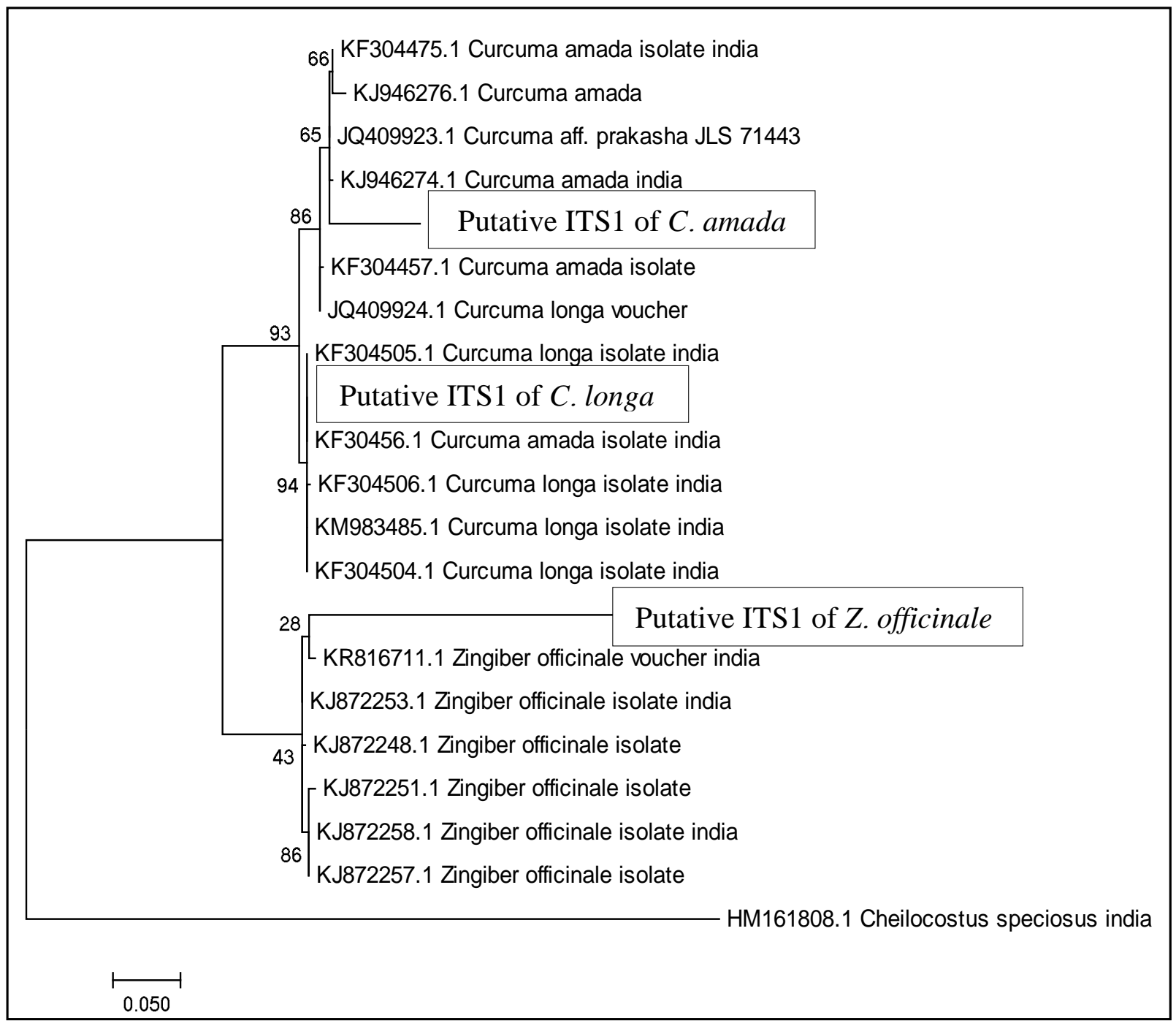

Figure 6: Molecular phylogenetic analysis by Maximum Likelihood method-bootstrap consensus tree. 


\section{Discussion}

Characterization is the description of a character or quality of an individual to determine the expression of highly heritable morphological, anatomical, chemical and genetic features which is important for their identification, phylogenetic and evolutionary studies (Vicente et al., 2005). Classification within a family and the delimitation of genera that have been done based on morphology and molecular characters can also be used to interpret phylogenetic relationships of a family (Kress et al., 2002).

According to the qualitative and quantitative morphological and anatomical characters of $Z$. officinale 'Local' and Z. officinale 'Chinese' were clustered into one clade with $100 \%$ similarity. In contrast in the chemical and genetic analysis, $Z$. officinale 'Local' was clustered with $Z$. officinale 'Rangun' in $100 \%$ similarity. Morphological characters are commonly used as a valuable tool in studying the development, population differentiation and systematics of plants nevertheless, morphological characters can be subjective (Vicente et al., 2005). Anatomical characters are more accurate compared to morphological characters as basic anatomical structures of a plant do not alter with environmental changes as morphological characters (Talip et al., 2003).

According to the loading plot plant height, stem diameter, length of rhizome internodes, leaf width, length of leaf petiole and length of internodes have shown correlation to the relationships of taxa when comparing with other characters hence, those characters can be recommended as more suitable for delimitation of taxa.

The three Zingiber cultivars have shown close phylogenetic relationship in their morphological and anatomical features with $64.64 \%$ similarity while $62.21 \%$ and $46.55 \%$ similarities have shown with respect to the genetic and chemical diversity respectively. The preliminary chemical screening was done only by comparing TLC profiles of phenolics and more precise relationships could have been derived if a detailed chemical analysis were performed.

A. calcarata has showed $56.70 \%$ similarity with the three $\mathrm{Z}$. officinale cultivars according to the morphological and anatomical features whereas $C$. longa and $A$. calcarata were clustered into a separate clade with $62.20 \%$ similarity according to their phenolic characters. However, in contrast to that $A$. calcarata has represented a separate evolutionary line according to the genetic information.

According to results in this study vascular bundle arrangement in members of Zingiberaceae leaf petiole is observed in ' $U$ ' shape indicating close relationship within the family and in agreement with the findings of Talip et al., (2003). Vascular strand arrangement of $C$. speciosus showed flat arc with rib traces in leaf petiole which indicate distant evolutionary relationship with Zingiberaceae taxa.

Stomata are usually classified with respect to the organization of the subsidiary cells and guard cells and similar configuration of subsidiary cells interprets the phylogenetic relationship. The stomata with paracytic subsidiary cells is considered as an evolutionary advanced character, which was found in most monocots (Priyadarshan, 2009). According to the results in this study $E$. cardomomum has showed paratetracytic stomatal type in which guard cells are surrounded by four subsidiary cells whereas the other species have shown paracytic stomatal type which subsidiary cells are organized parallel to long axis of stomatal pore in agreement with Priyadarshan (2009). 
Root cross sections of these studied taxa have showed a uniseriate epidermis with cortex consisted of an exoderm composed of 3-4 layers of cells while endoderm cell layer was clearly observed. The vascular cylinder of the root system formed as uniseriate pericycle and in agreement with Gevu et al., (2014).

The members of the order Zingiberales contain a high amount of polysaccharides and polyphenols which interfere in isolation procedures of total DNA and PCR based downstream applications. Therefore, removal of such contaminants is needed in developing suitable protocols (Devi et al., 2013). The midrib of the leaf had been removed, as it is the major source of carbohydrate contamination. Several researches have attempted to develop extraction methods by eliminating use of hazardous chemicals, equipment and expensive kits and has developed a CTAB based method for the extraction of high quality total DNA of different species of Zingiberales (Devi et al., 2013). However, that protocol was not successful in the present study for all the species studied therefore, few steps were changed. 1/10 volume of $7.5 \mathrm{M}$ ammonium acetate and 2 volumes of ice cold absolute ethanol were used instead of chilled isopropanol. The solution was then kept at $-20^{\circ} \mathrm{C}$ overnight instead of 30 minutes to extract sufficient amount and quality DNA from leaves.

\section{Conclusions}

Generally, many Zingiber taxa are producing flowers seasonally therefore, combination of morphological, anatomical and chemical characters of leaves and rhizomes can be useful for characterization of $Z$. officinale cultivars and Curcuma species in Sri Lanka during their initial developmental stages. Genetic characters are more reliable than morphological characters in characterization of these taxa. rDNA ITS region could be successfully used in Zingiber and Curcuma species delimitation. Derived phenetic and genetic relationships of these taxa would provide valuable information for plant breeders in their crop quality improvement programs.

\section{References}

Devi, K. D., Punvarani, K., Singh, N. S., Devi, H. S. (2013) An efficient protocol for total DNA extraction from the members of order Zingiberales-suitable for diverse PCR based downstream applications, SpringerPlus 2 (1): 669.

Gevu, K. V., Da Cunha, M., Barros, C. F., Pereira, S. M., Lima, H. R. P. (2014) Structural analvsis of subterranean organs in Zingiberaceae, Plant Systematics and Evolution 300 (5): 1089-1098.

Harborne, J. B. (1998) Phytochemical methods a guide to modern techniques of plant analysis, $2^{\text {nd }}$ edition. Chapman and Hall, New York, pp.92-110.

Iatoi, S. A., Kikuchi, A., Watanabe, K. N. (2007). Genetic diversity, cvtologv, and systematic and phylogenetic studies in Zingiberaceae, Genes, Genomes and Genomics 1 (1): 56-62.

Karunarathne, P., Yakandawala, D., Samaraweera, P. (2014) Taxonomy and Systematic Analysis of the Two Genera Alpinia and Amomum of the Family Zingiberaceae in Sri Lanka, Proceedings of International Forestry and Environment Symposium, 18.

Karunaratne, P. W. S. (2007). Phenetic relationships of the selected species of the family Zingiberaceae based on flavonoid characters. BSc. (Special) degree dissertation, Department of Botany, University of Kelaniya.

Kress W. J., Prince L. M., Willams, K. J. (2002). The phylogeny and new classification of the ginger 
(Zingiberaceae): evidence from molecular data, American Journal of Botany 89:1682-1696.

Priyadarshan, A. I. S. (2009). Phylogenetic relationships in selected monocots with basal angiosperms; morphometrics and flavonoid distribution. BSc. (Special) degree dissertation, Department of Botany, University of Kelaniya.

Talip, N., Hussin, K.H., Ibrahim, H. (2003). Comparative leaf anatomy of Alpinia species (Zingiberaceae) in Malavsia, Nordic Journal of Botany 23 (4): 463-483.

Uma, E., Muthukumar, T. (2014) Comparative root morphological anatomy of Zingiberaceae, Systematics and Biodiversity 12 (2): 195-209.

Vicente M., Guzmán F. A., Engels, J. (2005). Genetic characterization and its use in decision making for the conservation of crop germplasm, Biotechnology 5-7. 